\title{
Review
}

\section{Is adjuvant hysterectomy an option after radio-chemotherapy for locally advanced cervical cancer? A review.}

\author{
Cornelia Nitipir ${ }^{1,2}$, Cristina Orlov², Ana-Maria Popa ${ }^{2}$, Iulian Slavu1, Adrian Tulin ${ }^{1,3}$ \\ ${ }^{1}$ Carol Davila University of Medicine and Pharmacy, Bucharest, Romania \\ ${ }^{2}$ Elias University Emergency Hospital, Department of Oncology, Bucharest, Romania \\ ${ }^{3}$ Agrippa Ionescu Emergency Hospital, Department of General Surgery, Bucharest, Romania
}

\begin{abstract}
Objective. The purpose of this paper is to review the current concepts in the literature regarding the beneficial effects of adjuvant surgery after concurrent radio-chemotherapy for locally advanced cervical cancer.

Method. Research of the literature was performed using PubMed databases in order to find articles relevant to the central topic. The PICOS criteria were used to filter the results. The paper was then structured according to the PRISMA guideline.

Results. 50 individual papers were analyzed and sorted according to their relevance for the topic. 18 were classified as relevant. There was little agreement as to how beneficial adjuvant hysterectomy is in locally advanced cervical cancer after chemoradiotherapy. Some articles concluded that patients with a total clinical response after initial treatment might have superior progression-free survival and overall survival after surgery. 2 articles underlined the importance of accurate restaging after primary treatment. The lack of benefit for surgery in patients with bulky disease or persistent adenopathies was agreed upon in all cases.
\end{abstract}

Conclusions. The decision for adjuvant hysterectomy with or without lymphadenectomy is a controversial one and has to be personalized and taken in a multidisciplinary team.

\section{Keywords $\quad$ : cervical cancer, advanced cancer, adjuvant hysterectomy}

Highlights $\quad \checkmark \quad$ The decision for adjuvant hysterectomy for locally advanced cervical cancer (with or without lymphadenectomy) is still controversial and should be evaluated in a multidisciplinary team.

$\checkmark \quad$ The best candidates for surgery are the patients that for technical reasons could not receive the efficient total radiotherapy dose.

To cite this article: Nitipir C, Orlov C, Popa AM, Slavu I, Tulin A. Is adjuvant hysterectomy an option after radio-chemotherapy for locally advanced cervical cancer? A review. J Clin Invest Surg. 2018; 3(1): 9-13. DOI: 10.25083/2559.5555/31.913 


\section{Introduction}

Cervical cancer is a very important health issue nowadays, having the highest incidence in low-income countries. The most important factor in its incidence is the human papillomavirus- HPV infection. The prevalence of HPV infection in high incidence countries is $10-20 \%(1)$.

The most important histological subtype is squamous cell carcinoma- $80 \%$ followed by adenocarcinoma- 20\%. The incidence of adenocarcinoma of the cervix is ascending in the last 30 years, probably because screening methods for it are not as efficient as for squamous cell carcinoma $(2,3)$.

Advanced cervical cancer is usually defined as any stage higher than FIGO stage IIB (tumour with parametrial invasion). Radical hysterectomy with pelvic lymph node dissection is not an option for primary treatment for these patients and pelvic external beam radiotherapy (EBRT) and brachytherapy are standard options. Concurrent cisplatin chemotherapy should always be taken in consideration. The efficacy of adding concurrent chemotherapy cannot be doubted. However, just one-third of the patients have complete radiological in clinical response after radio-chemotherapy. The aim of this review is to present data in the literature regarding surgical efficacy in locally advanced cervical cancer patients after EBRT and brachytherapy with concomitant chemotherapy (4).

Research of the literature was performed using PubMed databases in order to find articles relevant to the central topic. The PICOS criteria were used to filter the results. The paper was then structured according to the PRISMA guideline.

Fifty individual papers were analyzed and sorted according to their relevance for the topic. 18 were classified as relevant. There was little agreement as to how beneficial adjuvant hysterectomy is in locally advanced cervical cancer after chemoradiotherapy. Some articles concluded that patients with a total clinical response after initial treatment might have superior progression-free survival and overall survival after surgery. Two articles underlined the importance of accurate restaging after primary treatment. The lack of benefit for surgery in patients with bulky disease or persistent nodal involvement was agreed upon in most clinical trials.

\section{Discussion}

- Surgery as primary treatment for cervical cancer Surgery is performed as primary treatment in stages I to $\mathrm{II}_{\mathrm{A}}$. Cone biopsy is an option in IA1 stage (5).
However, pelvic lymphodissection is mandatory if lymphovascular space invasion exists. In order to decide on the surgical procedure, the desire to preserve fertility is of utmost importance. If the patient is not a candidate for fertility-preserving procedures, then extrafascial hysterectomy is the right option. The decision for lymphodissection depends on LVSI in this case as well. In stage IA2 trachelectomy is the only option in order to preserve fertility (6).

In all the other cases in this stage hysterectomy with or without pelvic lymphadenectomy is the standard treatment. In stage IB to IIA fertility-sparing procedures are not an option. Radical hysterectomy with mandatory pelvic dissection and paraaortic lymph node sampling is the standard treatment. However, current guidelines underline that surgery as primary treatment is category I indication for stages IB1 and IIA and category IIB for stage IB2 and IIA2. For this last subgroup of patients, the preferred primary treatment is radiochemotherapy followed by brachytherapy (7).

- Primary treatment for locally advanced cervical cancer

Locally advanced cervical cancer is defined as any stage higher than IIB. Some experts consider stage IB2 and IIA2 locally advanced as well (8).

In the advanced setting, it is essential to establish whether nodal involvement is present because radiotherapy has to be tailored in consequence. The optimal methods to establish if nodal involvement exists are MRI and surgical staging. Surgical staging can identify microscopic nodal involvement and is superior to abdominopelvic MRI. PET-CT has higher sensibility and specificity for nodal involvement, but it not always available $(9,10)$.

Radiochemotherapy should always be chosen as primary treatment in these stages. EBRT will be planned using a CT scan. The EBRT plan should include the gross tumour volume, the parametria, the uterosacral ligaments, at least $3 \mathrm{~cm}$ of vaginal margin from the gross disease. If the patients are staged as node negative, then the radiation volume has to include the obturator, external and internal iliac nodes and presacral ones. If the patient is considered high risk the field has to be extended to the common iliac nodes. A total dose of $45 \mathrm{~Gy}$ has to be delivered to the lymph nodes. The total dose for the gross tumour volume has to be $85 \mathrm{~Gy}$ (as measured in point A). Point A is an anatomical point in the paracervical region, $2 \mathrm{~cm}$ above the cervical flange and $2 \mathrm{~cm}$ lateral to midline where highest doses of chemotherapy have to be delivered (11).

These high doses will be achieved with brachytherapy. The addition of cisplatin chemotherapy 
to this treatment is very efficient. The benefit of standard of therapy is available for advanced cervical concomitant cisplatin chemotherapy on overall survival cancer patients with a good response after and response rate is incontestable, making cisplatin chemoradiotherapy, the option of surgery is worth to be administration mandatory (12).

taken in consideration (14).

When the administration of these high doses of

Morice et al. published a trial on the same topic that radiation in locally advanced cervical cancer is not included only advanced cervical cancer patients with technically possible, and the total dose in point $\mathrm{A}$ is complete clinical and radiological response after lower, recent guidelines recommend adjuvant chemoradiotherapy. The study was underpowered by the hysterectomy. This, however, is a category 3 low number of patients. A total number of 61 patients recommendation (4).

- Evidence for adjuvant surgery after chemoradiotherapy in the advanced cervical cancer setting

One of the first clinical trials to investigate this option was the one by Perez et al. which included a total number of 425 patients with stage IB, IIA, IIB cervical cancer. All these patients were treated at first with chemoradiotherapy. The stage IB and IIA patients received a total dose to point A of 70-80 Gy, whereas the ones with bulky disease (tumour more than $5 \mathrm{~cm}$ diameter) or stage IIB were treated with total doses of $80-90$ Gy to point A. Overall survival at 5 years in patients IB2, IIA and IIB respectively was 61\%, 63\% and $69 \%$ in the radiochemotherapy alone group, and $60 \%, 72 \%$ and $65 \%$ in the group that also underwent surgery. A clear benefit on 10-year survival for the stage IIA patients that were also treated with adjuvant surgery was observed. $72 \%$ were alive at 10 years. However, statistical significance was disputable with p-value more than 0.5. Metastasis rate was lower in the radiochemotherapy alone group: for the IB, IIA, IIB subgroups $10 \%, 17 \%$ and $23 \%$ was the rate of metastasis in the radiochemotherapy alone subgroup, whereas $14 \%, 20 \%$ and $29 \%$ had metastases at the 5-year interval in the adjuvant surgery subgroup. This trial concluded that the outcomes are not different when adding surgery to radiochemotherapy for stage IB, IIA or IIB cervical cancer (13).

Another trial on this topic with an interesting design is the one of Legnevacue et al. published in 2011. It enrolled patients with advanced stage cervical cancer that received radiochemotherapy and had complete response on clinical gynecological examination and pelvic MRI or if the tumour was reduced by more than $50 \%$ in diameter. They were randomized for adjuvant hysterectomy or observation. Benefit on progressionfree survival was seen in the completion surgery group. The pelvic recurrence rate was lower in the surgery subgroup (46\% vs. 56\%) and the difference was statistically significant $(p<0.01)$. The relative risk of progression or death also favored the completion surgery group. The authors concluded, that although no were enrolled, of which 31 underwent surgery and 30 were observed. The surgical procedure consisted of extrafascial hysterectomy.

However, if adenopathies were identified intraoperatively, lymphadenectomy was done. 3-year progression-free survival was $72 \%$ in the observation group and $89 \%$ in the surgery one. OS at 3 years also favored the surgery group with $97 \%$ vs. $86 \%$. However, these differences were not backed up by statistical significance. One important aspect underlined by this trial was that the clinical and radiological complete response was confirmed by the pathology report in only one-third of patients. Therefore, in order to correctly restage patients after radiochemotherapy more sensible and specific methods like PET-CT have to be taken in consideration (15).

Darus et al. conducted a retrospective clinical trial that included only patients with stage IB that received primary radiochemotherapy and brachytherapy. The patients were divided into two subgroups: one that was assigned to observation alone and one that underwent extrafascial hysterectomy. 5-year OS was similar in the two subgroups (113.8 in the surgery subgroup and 113.7 in the radiochemotherapy and brachytherapy alone group). An interesting detail in this trial is that most of the patients that had recurrence in both groups were smokers (16).

One meta-analysis suggests that hysterectomy with or without lymphadenectomy after radiochemotherapy for cervical cancer has no benefit on OS, when reporting results for all the FIGO stage subgroups of patients. It may represent an option for patients with complete or good response to the first treatment. However, when bulky extra cervical disease or paraaortic lymph node involvement exist, the prognosis is worse. Surgical intervention, in this case, has no benefit and induces perioperative morbidity to the patient. However, there are few data on personalizing treatment for each histologic subtype: especially adenocarcinoma vs. squamous cell carcinoma (17). 
Some trials consider adjuvant hysterectomy beneficial for patients with the macroscopic persistent disease after radiochemotherapy (18).

A trial of Houveraeghel et al. describes clear benefit on survival for radical hysterectomy after chemoradiotherapy for patients with the macroscopic persistent disease. However, none of the cases could have been classified as bulky dease after initial therapy. None of them has suspicious local lymphnodes. The reported 5-year OS in the surgery group was 64.9 percent for stages higher than IIB (19).

\section{Conclusions}

The decision to operate after primary external beam radiochemotherapy and brachytherapy was recently introduced in current guidelines for selected cases. The best candidates for surgery are the patients that for technical reasons could not receive the efficient total radiotherapy dose. Another subgroup of patients that could benefit from this approach are the ones with radiologic and clinical complete response. No benefit was seen for patients with the persistent bulky disease after primary treatment or for those with positive lymph nodes after treatment. Therefore, the decision for adjuvant hysterectomy with or without lymphadenectomy is a controversial one and has to be taken in a multidisciplinary team.

\section{References}

1. Wang SS, Carreon JD, Gomes SL, Devessa SS. Cervical cancer incidence among 6 asian ethnic groups in the United States, 1996-2004. Cancer 2010; 116(4): 949-56. PMID: 20029972, DOI: $10.1002 /$ cncr. 24843

2. Kamphuis EI, Naber SK, Danhof NA, Habbema JDF, de Groot CJM, Mol BWJ. Effect of Cervical Cancer Screening Programs on Preterm Birth: A Decision and Cost-Effectiveness Analysis. Obstet Gynecol. 2017; 130(6): 1207-17. PMID: 29112652 , DOI: 10.1097/AOG.0000000000002366

3. Jemal A, Bray F, Center MM, Ferlay J, Ward E, Forman D. Global cancer statistics. CA Cancer $J$ Clin. 2011; 61(2): 69-90. PMID: 21296855, DOI: 10.3322/caac. 20107

4. NCCN Clinical Practice Guidelines in Oncology (NCCN Giudelines)- Cervical cancer. Version 1.2018, October 2017. Accessed on 24.02.2018

5. Ramirez PT, Pareja R, Rendon GJ, Millan C, Frumovitz M, Schmeler KM. Management of low risk early stage cervical cancer: should conization, simple trachelectomy or simple hysterectomy replace radical surgery as the new standard of care? Gynecol Oncol. 2014; 132(1): 254-9. PMID: 24041877, DOI: 10.1016/j.ygyno.2013.09.004

6. Cao DY, Yang Jx, Wu XH, Chen YL, Li L, Liu KJ, Cui MH, Xie X, Wu YM, Kong BH, Zhu GH, Xiang Y, Lang JH, Shen K; China Gynecologic Oncology Group. Comparisons of vaginal and abdominal radical trachelectomy for early stage cervical cancer: preliminary results of a multi-center research in China. Br J Cancer. 2013; 109(11): 2778-82. PMID: 24169350,

DOI: $10.1038 /$ bjc. 2013.656

7. Cibula D, Abu-Rustum NR, Benedetti- Panici P, Köhler C, Raspagliesi F, Querleu D, Morrow CP. New classification system of radical hysterectomy: emphasis of a three dimensional anatomic template for parametrial resection. Gynecol Oncol. 2011; 122(2): 264-8. PMID: 21592548, DOI: 10.1016/j.ygyno.2011.04.029

8. Pecorelli S, Zigliani L, Odicino F. Revised FIGO staging for carcinoma of the cervix. Int J Gynecol Obstet. 2009; 105(2): 107-8. PMID: 19342051, DOI: 10.1016/j.ijgo.2009.02.009

9. Gold MA, Tian C, Whitney CW, Rose PG, Lanciano R. Surgical vs. radiographic determination of para-aortic lymphnode metastases before chemoradiotion for locally advanced cervical carcinoma: a Gynecologic Oncology Group Study. Cancer. 2008; 112(9): 1954-63. PMID: 18338811, DOI: 10.1002/cncr.23400

10. Patel S, Liyanage SH, Sahdev A, Rockall AG, Reznek RH. Imaging of endometrial and cervical cancer. Insights Imaging. 2010; 1(5-6): 309-28. PMID: 22347925, DOI: 10.1007/s13244-0100042-7

11. Yoshida K, Jastaniyah N, Sturdza A, Lindegaard J, Segedin B, Mahantshetty U, Rai B, JürgenliemkSchulz I, Haie-Meder C, Sasaki R, Pötter R. Assesment of Parametrial Response by Growth Pattern in Patients with IIB and IIIB Stage Cervical Cancer. Analysis of Patients from a Prospective, Multicenter Trial (EMBRACE). Int $J$ Radioat Oncol Biol Phys. 2015; 93(4): 788-96. PMID: 26530747, DOI: 10.1016/j.ijrobp.2015.08.007

12. Gaffney DK, Erickson Wittmann BA, Jhingran A, Mayr NA, Puthawala AA, Moore D, Rao GG, Small W Jr, Varia MA, Wolfson AH, Yashar CM, Yuh W, Cardenes HR. ACR Appropriateness Criteria (R) on Advanced Cervical Cancer Expert panel on Radiation Oncology-Gynecology. Int $J$ Radiat 
Oncol Biol Phys. 2011; 81(3): 609-14. PMID: 21215531, DOI: 10.1016/j.ijrobp.2010.11.005

13. Perez CA, Grigsby PW, Camel HM, Galakatos AE, Mutch D, Lockett MA. Irradiation alone or combined with surgery in stage IB, IIA, and IIB carcinoma of uterine cervix: update of a nonrandomized comparison. Int J Radiat Oncol Biol Phys. 1995; 31(4): 703-16. PMID: 7860381

14. Leguevaque P, Motton S, Delannes M, Querleu D, Soule-Tholy M, Tap G, Houvenaeghel G. Completion surgery or not after concurrent chemoradiotherapy for locally advanced cervical cancer? Eur J Obstet Gynecol Reprod Biol. 2011; 155(2): 188-92. PMID: 21232839, DOI: 10.1016/j.ejogrb.2010.11.016

15. Morice $\mathrm{P}$, Rouanet $\mathrm{P}$, Rey A, Romestaing $\mathrm{P}$, Houvenaeghel G, Boulanger JC, Leveque J, Cowen D, Mathevet P, Malhaire JP, Magnin G, Fondrinier E, Berille J, Haie-Meder C. Results of the GYNECO 02 study, an FNCLCC phase III trial comparing hysterectomy with no hysterectomy in patients with a (clinical and radiological) complete response after chemoradiation therapy for stage IB2 or II cervical cancer. Oncologist. 2012; 17(1):64-71. PMID: 22234626,

DOI: 10.1634/theoncologist.2011-0276
16. Darus CJ, Callahan MB, Nguyen QN, Pastore LM, Schneider BF, Rice LW, Jazaeri AA. Chemoradiation with and without adjuvant extrafascial hysterectomy for IB2 cervical carcinoma. Int J Gynecol Cancer. 2008; 18(4): 730-5. PMID: 17949426, DOI: 10.1111/j.15251438.2007.01095.x

17. Shi D, Liang Z, Zhang C, Zhang H, Liu X. The effect of surgery on the survival status of patients with locally advanced cervical cancer after radiotherapy/chemoradiotherapy: a meta-analysis, BMC Cancer. (2018); 18(1): 308. PMID: 29558900, DOI: 10.1186/s12885-018-4232-x

18. Ota $\mathrm{T}$, Takeshima $\mathrm{N}$, Tabata $\mathrm{T}$, Hasumi $\mathrm{K}$, Takizawa K. Adjuvant hysterectomy for treatment of residual disease in patients with cervical cancer treated with radiation therapy. $\mathrm{Br} J$ Cancer. 2008; 99(8): 1216-20. PMID: 18854823, DOI: 10.1038/sj.bjc.6604619

19. Houvenaeghel G, Lelievre L, Buttarelli M, Jacquemier J, Carcopino X, Viens P, GonzagueCasabianca L. Contribution of surgery in patients with bulky residual disease after chemoradiation for advanced cervical carcinoma. Eur J Surg Oncol. 2007; 33(4): 498-503. PMID: 17156969, DOI: 10.1016/j.ejso.2006.10.011 